\title{
Energy efficient anti-collision algorithm for the RFID networks
}

\author{
Murukesan Loganathan, Thennarasan Sabapathy, Mohamed Elshaikh, Mohamed Nasrun Osman, \\ Rosemizi Abd Rahim \\ School of Computer and Communication Engineering, University Malaysia Perlis, Malaysia
}

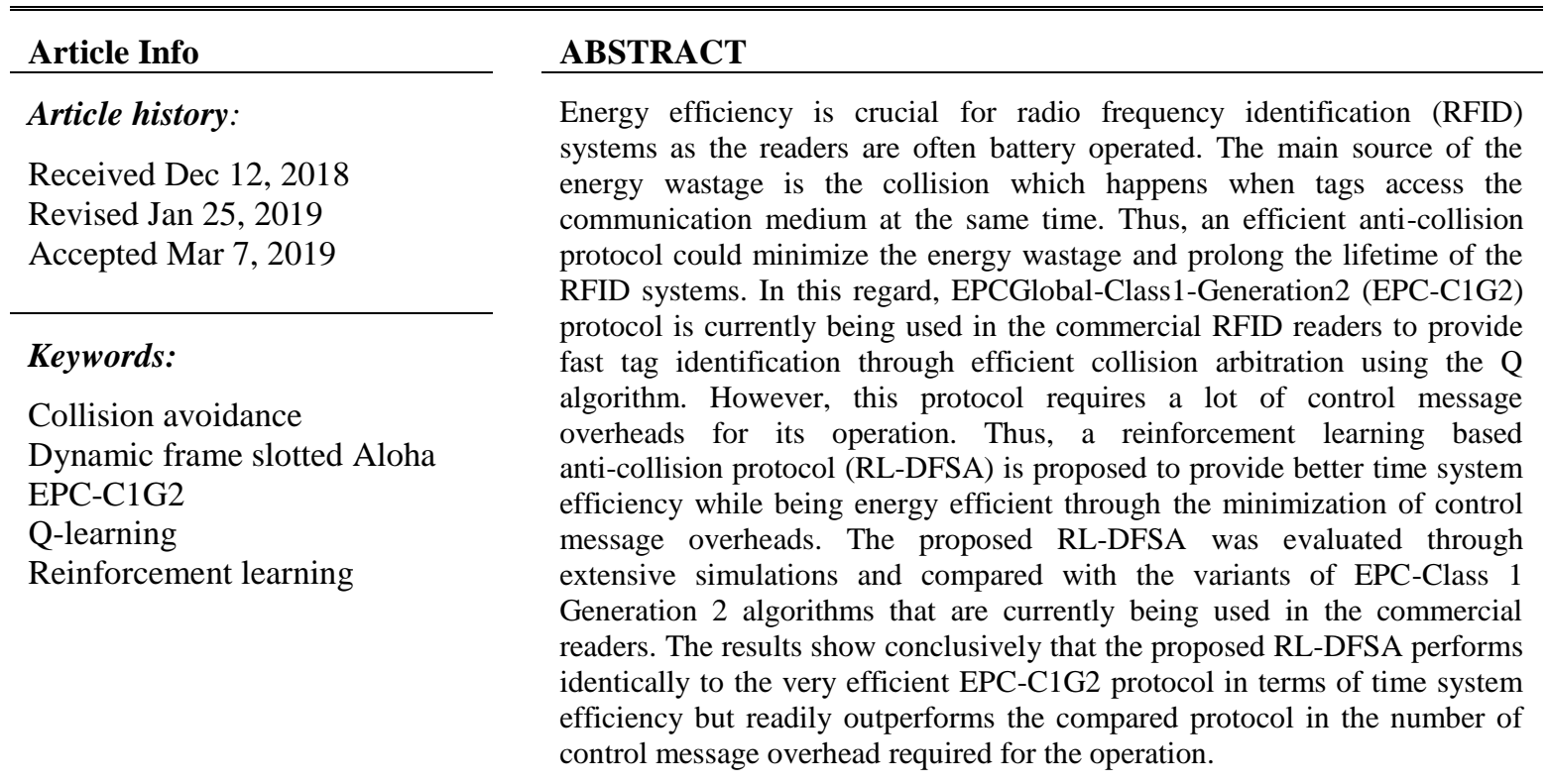

Copyright (ㅇ 2019 Institute of Advanced Engineering and Science. All rights reserved.

\section{Corresponding Author:}

Murukesan Loganathan,

School of Computer and Communication Engineering,

University Malaysia Perlis (UniMAP),

Campus Pauh Putra, 02600 Arau, Perlis, Malaysia.

Email: murukesan.loganathan23@gmail.com

\section{INTRODUCTION}

Radio frequency identification (RFID) is a technology that uses radio waves for the purpose of identifying a large number of goods in a swift manner. This technology has widespread acceptance in various fields of applications such as inventory management, logistic, retailing and dairy farms [1]. A typical RFID setup has at least one reader with numerous RFID tags. RFID tags are classified into passive or active tags depending on the availability of a power source on them. A passive tag gets its power from the RF signal of the reader while an active tag has its own battery. Thus, the communication range of an active tag is much longer as compared to the passive tags. Despite having clear disadvantage in communication distance, passive tags are largely favored due to the advantages of low deployment cost and longer lifetime. Hence, we consider only an RFID system with a reader and numerous passive tags in this paper. Also, reader-to-reader interference is out of the scope of this paper.

RFID tags communicate with the reader using a shared communication channel. Thus, collisions are inevitable when multiple tags try to communicate their ID at the same time to the reader [2]. This problem is particularly challenging since medium access schemes like frequency division multiple access (FDMA) or code division multiple access (CDMA) are cannot be implemented due to the computational limitations of the passive tags. Therefore, the burden of alleviating collisions in the network falls on the reader. There are 
numerous collision arbitration protocols available in the literature for the RFID systems. Among those protocols, Framed Slotted Aloha (FSA)-based protocols are popular and are being used extensively in RFID standards due to the simplicity and good performance [3,4]. FSA protocol is probabilistic in nature in which tags select a random slot to send their ID. Besides, in FSA, tags won't check the channel to see whether it is busy or free before start transmitting their own ID. The usage of random transmission strategy with time slots reduces the collision probability in this protocol. Current RFID standard, EPCGlobal Class 1 Generation 2 (EPC-C1G2) uses a variant of FSA for its operation [3].

The EPC-C1G2 protocol is used extensively in current generation commercial RFID devices since it is the defacto standard of the industry. Simplicity and high throughput of the algorithm helped in its rapid adoption in the industry. However, EPC-C1G2 generates a high amount of control messages which increases the energy consumption [5]. Despite the shortcoming, EPC-C1G2 is still favored as an efficient anti-collision algorithm (ACA) thanks to its high time system efficiency (TSE) as shown in Figure 1. Therefore, in this paper, we present an efficient ACA namely, reinforcement learning based dynamic frame slotted Aloha (RL-DFSA) which can perform as good as EPC-C1G2 protocol without the need for large number of control message exchanges. The performance of the proposed algorithm (RL-DFSA) was evaluated using Monte-Carlo simulations (5000 iterations) and compared with algorithms that are currently being used in commercial settings. RL-DFSA is very efficient in identifying tags as exhibited by its high TSE metric and requires one order of magnitude lesser message exchanges than that of the best performing algorithm in the commercial readers.

The remainder of this paper is organized as follows. Section 2 discusses the current RFID standard and related works. In Section 3, the complete methodology of the proposed RFID anti-collision protocol is presented in detail. Section 4 presents results and discussion of the proposed protocol in relation to selected protocols from the literature. Finally, the paper concludes with concluding remarks and future works in Section 5.

\section{BACKGROUND INFORMATION AND RELATED WORKS}

\subsection{Primer on FSA and Q-Algorithm}

In FSA, a frame is divided into slots of same length [6]. At the beginning of each frame, interrogator or reader broadcasts the frame size to the tags. The tags then select a slot randomly and send the ID information to the reader in that slot. Due to this random slot selection policy, excessive collisions are bound to happen depending on the tag population if a non-optimal frame size is selected by the reader. The average throughput, $U$ of FSA for $\mathrm{N}$ tag population and frame size $L$ is:

$$
\mathrm{U}=\mathrm{N}\left(1-\frac{1}{\mathrm{~L}}\right)^{\mathrm{N}-1}
$$

and the normalized throughput, $\mathrm{U}_{\text {norm }}$ is given by:

$$
\mathrm{U}_{\text {norm }}=\frac{\mathrm{N}}{\mathrm{L}}\left(1-\frac{1}{\mathrm{~L}}\right)^{\mathrm{N}-1}
$$

The normalized throughput is maximized when $L=N$. However, readers are not privy of the tag population and FSA has a fixed frame size. Due to these limitations, a variant of FSA called dynamic frame slotted aloha (DFSA) which adapts frames dynamically based on the backlog tag estimation was proposed in the literature [7]. However, its throughput drops significantly as the number of tags increases. Thus, Q-algorithm, a variant of DFSA was employed instead in the current generation RFID standard.

Q algorithm operates using two parameters, namely, a floating-point parameter, $Q_{f p}$ and $c_{q}$. The round $Q_{f p}$ value is used to set the frame size, $L$ and the $c_{q}$ is used to increase or decrease the $Q_{f p}$ value in the event of collision or empty slots, respectively. An interrogation process is initiated by the reader with th broadcast of a Query command which contains the frame size. Upon receiving this command, tags generate a random number in the range of $0-2^{Q-1}$ and set their counter equal to the generated value. Then, the reader interrogates each slot of the frame one by one using the Query_repeat command. For each Query_repeat command, tags decrease their counter by one. Tag with counter equals to zero transmits its ID information to the reader. However, if there are more than one tag with counter equals zero for current slot, a collision would be detected by the reader. Consequently, the $Q_{f p}$ is increased by some pre-determined $c_{q}$ value. In the case of empty slot, $Q_{f p}$ would be decreased by the same $c_{q}$ value. The round $Q_{f p}$ value would be updated continuously for each slot until a change is detected upon which the reader would exit the current frame and broadcasts new frame size using Query adjust command. This process repeats until all tags are identified. The standard limits the round $Q_{f p}$ value in the range between 0 to 15 for delay concerns. Besides, 
reader has the autonomy to decide whether to exit the current frame or continue interrogating it even when the round $Q_{f p}$ value had changed.

One unique feature of the EPC-C1G2 algorithm is that it has different time durations for success, collision and empty slots as per the standard. Thus, the claim that the throughput of FSA maximized when $L=N$ is not applicable even though EPC-C1G2 is a variant of FSA. This has been verified analytically by [8] and the optimal frame size, $L$ for EPC-C1G2 was calculated as:

$$
\mathrm{L}=1.46 \times \mathrm{N}-1
$$

where, $N-1$ is the contending tag population.

Q-algorithm has several drawbacks as follow. The initial selection of the $Q$ value affects its performance significantly. But the reader has no means to know the population of tags in the network a priori to set the $Q$ value appropriately. Besides, $Q$ adjustment strategy using $c_{q}$ produces excessive protocol overheads and also performs poorly in dense tag environment.

\subsection{Related works}

The limitations of the FSA propelled numerous research efforts which resulted in dynamic frame length Aloha, also known as dynamic frame slotted Aloha (DFSA) [7]. In DFSA, frame size is adjusted at each communication interval based on the estimated number of backlog tags given by:

$$
\mathrm{L}=2.39 \times \mathrm{C}
$$

where, $C$ is the number of collided slots in the previous frame. DFSA achieves normalized throughput of 0.426 as compared to 0.368 in slotted Aloha. Hence, it is clear that the performance of FSA can be further improved by dynamically adapting the frame size which in turn depends on the prediction accuracy of the number of unidentified tags.

Vogt developed unidentified or backlog tag estimation based on number of empty (E), collision (C) and success (S) slots in the previous frame [9]. The author selected the $N$ which minimize the distance of two vectors,

$$
\varepsilon_{\mathrm{vd}}=\min \left|\left(\begin{array}{c}
\mathrm{a}_{0} \\
\mathrm{a}_{1} \\
\mathrm{a}_{\mathrm{m}}
\end{array}\right)-\left(\begin{array}{c}
\mathrm{E} \\
\mathrm{s} \\
\mathrm{C}
\end{array}\right)\right|
$$

The expected values of empty, success and collision slots are:

$$
\begin{aligned}
& a_{0}=L\left(1-\frac{1}{L}\right)^{N} \\
& a_{1}=N\left(1-\frac{1}{L}\right)^{N-1} \\
& a_{m}=1-a_{0}-a_{1}
\end{aligned}
$$

Nevertheless, it is intuitively clear that an ACA should over allocate slots at the beginning of the interrogation round and reduce it gradually over the time to reduce the delay. However, existing algorithms in the literature use a static policy in which a static constant is used such as in (4) to set the frame size regardless of the fact that number of unidentified tags reduces over the time. Thus, in this work, we used reinforcement learning to address both the overallocation and static contant issues by learning an optimal policy.

\section{METHODOLOGY}

In this section, we present a novel and efficient frame adaptation method called reinforcement learning based dynamic frame slotted Aloha (RL-DFSA). We used the Q-learning algorithm since it is known to be one of the most effective and popular algorithms to find an optimal policy in the absence of transition probability and reward function [10].

Q-learning is a model-free reinforcement learning algorithm which learns by interacting with the environment and receiving Q-value for the state-action pair. The Q-value denotes the preference of taking an action over all other available actions when the system is at a certain state. Formally, for each state $s_{t} \in S$ and action $a_{t} \in A$ we define Q-value by: 


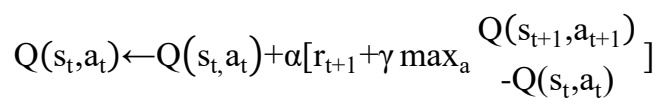

where, $\alpha$ is the learning rate, $\gamma$ is the discount factor and $r_{t+1}$ is the delayed reward.

We now describe how Q-learning can be used for adapting framed slotted Aloha protocol dynamically in RFID systems. We are well aware of the computational and space constraints of a typical RFID reader. However, there are numerous very powerful current generation RFID readers such as GAORFID, RapidRadio etc. which have ARM processor and memory card supports [11, 12]. We envision our algorithm to run on these powerful readers to improve their tag reading efficiency.

The proposed RL-DFSA has two phases, namely, learning (exploration + exploitation) and testing (exploitation only) due to the technical difficulty in running both the learning and testing, concurrently. For 1000 number of tags, RL-DFSA requires 20,000 iterations ( 12 minutes) to converge to an optimal policy. Therefore, learning and testing phases were conducted separately for time concerns. Readers are reminded that RL-DFSA can run online on current generation high-end RFID readers since the algorithm only needs to run as long as there are tags to be read unlike 5000 iterations for each tag population as in the current simulations. Moreover, the slow convergence of the algorithm is due to the stochastic nature of our application and Q-learning itself is slow as rightly observed by [13].

Frame Slotted Aloha is considered in this work and Q-learning is used to capture the learning experience. A frame comprises multiple slots in which the reader communicates with nearby tags. Q-learning is applied on the reader side as the tags are very primitive for the needed computation. A reader would initialize an interrogation round and continuously adapt the frame size until all the tags in the range are identified. However, the reader is not privy of the tag population as in the real-world application. Thus, the reader needs to estimate the contending tags population and adapt the frame size based on the available information such as collisions, empty and successful slots in the previous frame. There are numerous tag estimation and frame size adaptation methods available in the literature as reviewed in Section 2 . In this work, Q-learning was used to solve both the tag estimation and frame size adaptation problems of RFID system.

It is evident from Section 2 that the frame size should be 1.46 times larger than the contending tags population. Thus, the focus shifted from frame size estimation to number of tags estimation since the efficiency of the algorithm now rests solely on the accuracy of the tag estimation algorithm. The number of tags transmitting at the same slot can vary according to the availability of number of slots and tag population. However, the lower bound for the number of tags in a collision slot is two. In this work, we defined the actions for Q-learning based on this rational intuition. Initially, eleven actions were defined as follow:

Action $1=1.46 \times 2.0 \times$ number_of_collision

Action $2=1.46 \times 2.2 \times$ number_of_collision

and so on until,

Action $11=1.46 \times 4.0 \times$ number_of_collision

The number of actions was limited at eleven since increasing it indefinitely would increase the computational and space complexities, exponentially. The state of the learning agent is defined as the number of collisions in the previous frame. The goal of the learning agent is to reduce the number of collisions to zero. The agent is assisted in its task through a reward function. Reward function for this work is defined using the collision ratio ( $\left.\frac{\text { number_of_collision }}{\text { frame size }}\right)$ as follows:

reward $=\left\{\begin{array}{c}-1, \text { ratio }>0 \text { and }<\frac{1}{4} \\ -2, \text { ratio } \geq \frac{1}{4} \text { and }<\frac{1}{2} \\ -4, \text { ratio } \geq \frac{1}{2} \text { and }<\frac{3}{4} \\ -8, \quad \text { ratio } \geq \frac{3}{4}\end{array}\right.$

An initial study was conducted to identify dominant actions based on the cumulative sum of the Q values. Dominant actions were characterized by the relatively high cumulative sum of the Q-values. We ranked the cumulative sum from highest to lowest and top 3 actions were identified as dominant. Only three actions (4, 2 and 1) were selected since the difference in cumulative sum of the Q-values between the third and following actions were insignificant. Using the optimal actions identified in the initial study, another 
simulation was performed to acquire the optimal policy and Q-matrix for a tag population of 1000. The parameters of RL-DFSA algorithm for the initial study are presented in Table 1. The initial state of the agent can be any arbitrary value except one since state one is the goal state. The timing parameters given in Table 2 were used for all our simulations. The pseudocode of RL-DFSA is presented in Algorithm 1. In the testing phase, the learned Q-matrix was used to select an optimal action during each state.

Table 1. RL-DFSA parameter for the initial study

\begin{tabular}{lc}
\hline \multicolumn{1}{c}{ Parameter } & Value \\
\hline Initial state & 2 \\
Action & 11 \\
Learning rate, $\alpha$ & 0.1 \\
Discount rate, $\gamma$ & 0.9 \\
Exploration, $\varepsilon$ & 0.3 \\
Epsilon decay rate & 0.99971 \\
Maximum iteration & 30,000 \\
Number of tags & 1000 \\
Initial frame size & 16 \\
\hline
\end{tabular}

Table 2. EPC-Gen2 reader interrogation parameters [14]

\begin{tabular}{cccc}
\hline Parameter & Duration & Parameter & Duration \\
\hline Tari & $6.5 \mu \mathrm{s}$ & Tari & $6.5 \mu \mathrm{s}$ \\
RTcal & $16.25 \mu \mathrm{s}$ & RTcal & $16.25 \mu \mathrm{s}$ \\
BLF & $394 \mathrm{kHz}$ & BLF & $394 \mathrm{kHz}$ \\
$\mathrm{T}_{1}$ & $20.84 \mu \mathrm{s}$ & $\mathrm{T}_{1}$ & $20.84 \mu \mathrm{s}$ \\
$\mathrm{T}_{2}$ & $7.61 \mu \mathrm{s}$ & $\mathrm{T}_{2}$ & $7.61 \mu \mathrm{s}$ \\
$\mathrm{TRext}_{\mathrm{M}}$ & 1 & $\mathrm{TRext}$ & 1 \\
$\mathrm{M}$ & 1 & $\mathrm{M}$ & 1 \\
$\mathrm{~T}_{\mathrm{RN} 16}$ & $126.9 \mu \mathrm{s}$ & $\mathrm{T}_{\mathrm{RN} 16}$ & $126.9 \mu \mathrm{s}$ \\
$\mathrm{T}_{\mathrm{EPC}}$ & $695.43 \mu \mathrm{s}$ & $\mathrm{T}_{\mathrm{EPC}}$ & $695.43 \mu \mathrm{s}$ \\
$\mathrm{T}_{3}$ & $25.381 \mu \mathrm{s}$ & & \\
\hline
\end{tabular}

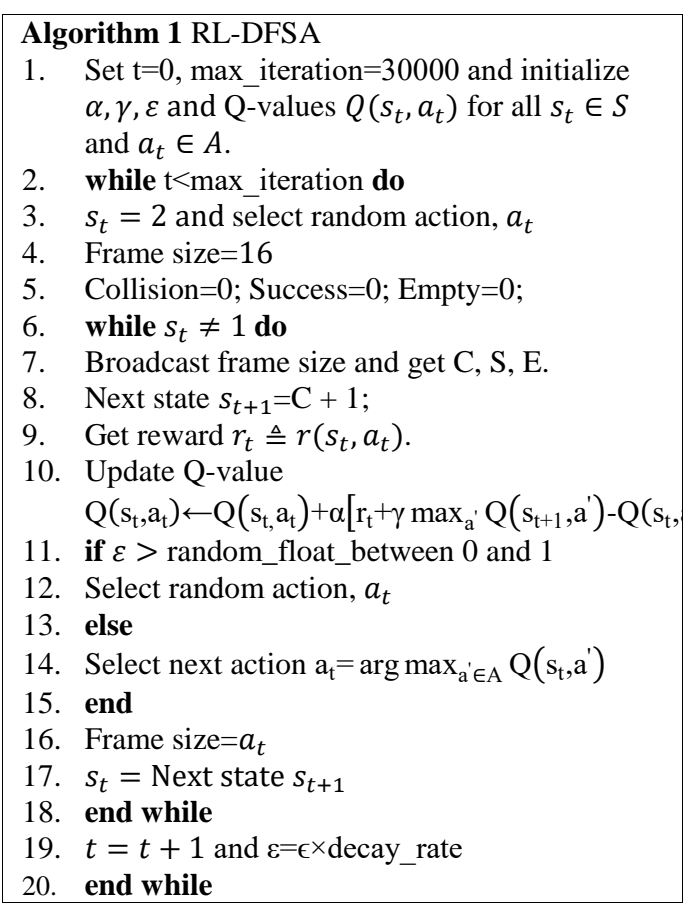

\section{SIMULATION RESULTS AND DISCUSSION}

The collision arbitration performance of the proposed algorithm and the reference algorithms including EPC-Q-Slot, EPC-Q-Frame [15], EPC-Fixed [15] and Ideal was compared through extensive Monte Carlo simulations under Matlab software environment. All the EPC algorithms are variants of EPC-C1G2 algorithm which was discussed in Section 2. EPC-Q-Slot algorithm simulates the scenario where the reader immediately issues the Query_adjust command as soon as a variation in round $\left(\mathrm{Q}_{\mathrm{fp}}\right)$ value is detected. In contrast, EPC-Q-Frame simulates the scenario when the reader only issues a Query_adjust command at the beginning of a new frame even though the round $\left(\mathrm{Q}_{\mathrm{fp}}\right)$ value had changed midframe. Besides, EPC-Fixed algorithm simulates the commercial readers with fixed frame size. Finally, the Ideal algorithm was used as an upper bound of performance which can be achieved by an algorithm which knows the tag population a priori. Two metrics, namely, the time system efficiency and the average number of frames are considered to evaluate the performance of RL-DFSA.

a. Time system efficiency (TSE) [8]-> This metric gives the percentage of time successfully spend in identifying tags. It is calculated as follow:

$$
\mathrm{TSE}=\frac{\text { Success } \times \mathrm{T}_{\mathrm{S}}}{\text { Success } \times \mathrm{T}_{\mathrm{S}}+\text { Empty } \times \mathrm{T}_{\mathrm{e}}+\text { Collision } \times \mathrm{T}_{\mathrm{c}}}
$$

where, Success, Collision, and Empty denote the number of successful, collided and empty slots in the frame, respectively. $T_{s}, T_{e}$, and $T_{c}$ are the duration of successful, empty and collision slots, respectively.

b. Average number of frames per round $\rightarrow$ This metric gives us an average number of frames required by the reader for each interrogation round.

The primary time parameters for the simulations were obtained from [14] and are presented in Table 2. Besides, the simulation scenario was divided into two-sparse (10-100 tags) and dense (100-1000 tags) environments-for an easier interpretation of the results. The initial simulation parameters of all the algorithms are given in Table 3. The performance of RL-DFSA in terms of TSE was evaluated by comparing it with the other four algorithms for a various number of tags as shown in Figure 1. 
Table 3. Initial parameters for the algorithms

\begin{tabular}{ccc}
\hline Algorithm & Parameter & Value \\
\hline \multirow{2}{*}{ EPC-Q-Slot } & $\mathrm{Q}$ & 4 \\
& $\mathrm{c}_{\mathrm{q}}$ & 0.3 \\
EPC-Q-Frame & $\mathrm{Q}$ & 4 \\
& $\mathrm{c}_{\mathrm{q}}$ & 0.3 \\
\multirow{2}{*}{ EPC-Fixed } & $\mathrm{Q}$ (Sparse) & 4 \\
& $\mathrm{Q}$ (Dense) & 7 \\
\multirow{2}{*}{ Ideal } & $\mathrm{c}_{\mathrm{q}}$ & 0.3 \\
& Initial Frame Size & 16 \\
\multirow{2}{*}{ RL-DFSA } & Subsequent Frame & $1.46 \times$ no. of remaining_tags \\
& Initial Frame Size & 16 \\
& Subsequent Frame & Based on the learned policy \\
\hline
\end{tabular}
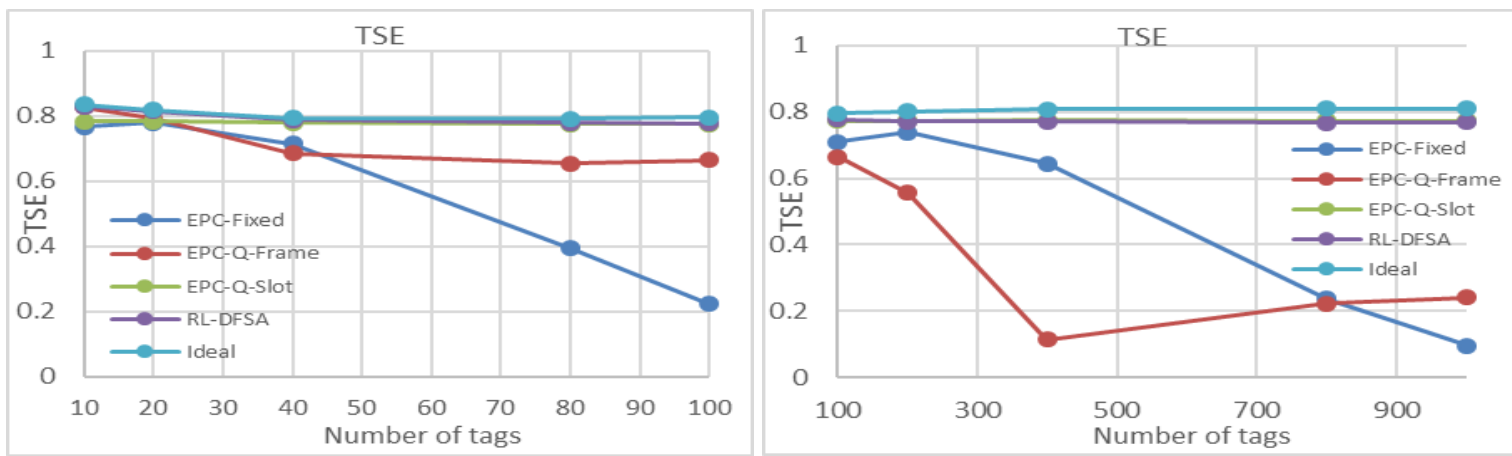

Figure 1. Time system efficiency of the algorithms for a different number of tags

The Ideal algorithm serves as an upper bound of performance that can be achieved when the reader knows exactly the number of tags to be identified and set the frame-size to 1.46 times the contending tag population. In both the sparse and dense tag environments, EPC-Q-Slot performs identically to RL-DFSA as we can see in Figure 1. Both these algorithms achieve near-optimal performances thanks to their efficient frame size adaptation mechanism. In the case of EPC-Q-Slot, the ability to adjust the frame size as soon as the round $\mathrm{Q}_{\mathrm{fp}}$ changes provide it with the needed dynamic adaptation for any number of tag population. On the other hand, RL-DFSA selects an optimal frame size for each iteration based on the received positive or negative feedback for its decision on the previous frame. This ability to adjust its action based on rewards and punishment makes it suitable for dynamic environments such as the RFID systems. In contrast, both the EPC-Q-Frame and EPC-Fixed algorithms are unable to cope up with the dynamic tag population as can be seen from their unstable and inferior TSE performance. Besides, EPC-Fixed performed the worst since dynamic frame size adaptation is absent when the number of tags changes. Overall, RL-DFSA is $6.3 \%-250 \%$, $0.4 \%-18.6 \%$ and $0.4 \%-5.7 \%$ better at TSE for sparse tag environment as compared to EPC-Fixed, EPC-Q-Frame and EPC-Q-Slot algorithms, respectively. Also, for dense tag environment, RL-DFSA performs $5.3 \%-707.4 \%$ and $17 \%-578.8 \%$ better as compared to EPC-Fixed and EPC-Q-Frame algorithms, respectively. The performance of RL-DFSA and EPC-Q-Slot algorithms is identical in both the sparse and dense tag environments as far as the TSE metric is concerned:

It is evident from the literature that the radio transmitter module of sensor nodes consumes almost three times more energy as compared to the node's microcontroller [16]. So, it is perfectly logical to opt for longer computation if it can reduce the required number of radio transmissions. In our case, RL-DFSA requires a much larger number of computations as compared to the other four algorithms since it needs to update the state-reward matrix using (9) after each transmission. However, the tradeoff between the energy required for communication and computation becomes irrelevant if the performance of the proposed algorithm is subpar. Incidentally, RL-DFSA performs identically to EPC-Q-Slot which is the best performing commercial algorithm while requiring an order of magnitude lower number of frames as presented in Figure 2. The difference in the number of frames is much more pronounced in the dense tag environment. Through reducing the number of Query_adjust commands by selecting optimal frame in each round, RL-DFSA improves energy efficiency. Meanwhile, EPC-Fixed performed worse as compared to EPC-Q-Slot in sparse tag environment since the frame size of 16 is not enough to accommodate a larger number of tags. However, its performance increases in dense tag environments since the new frame size is 128 . Generally, algorithms which adapt their frame size following a frame-by-frame adjustment strategy require a smaller number of control messages as compared to the algorithms with slot-by-slot adjustment techniques. The reason for using 
slot-by-slot adjustment strategy in commercial readers is mainly due to its high TSE performance which is hardly achievable by normal frame-by-frame algorithms as shown in Figure 1. However, RL-DFSA can guarantee a similar TSE performance that of the EPC-Q-Slot without the need for high communication cost due to the issuance of numerous Query_adjust commands. This high efficiency of the proposed algorithm is due to its ability to learn an optimal policy even in a dynamic environment such as the RFID systems.
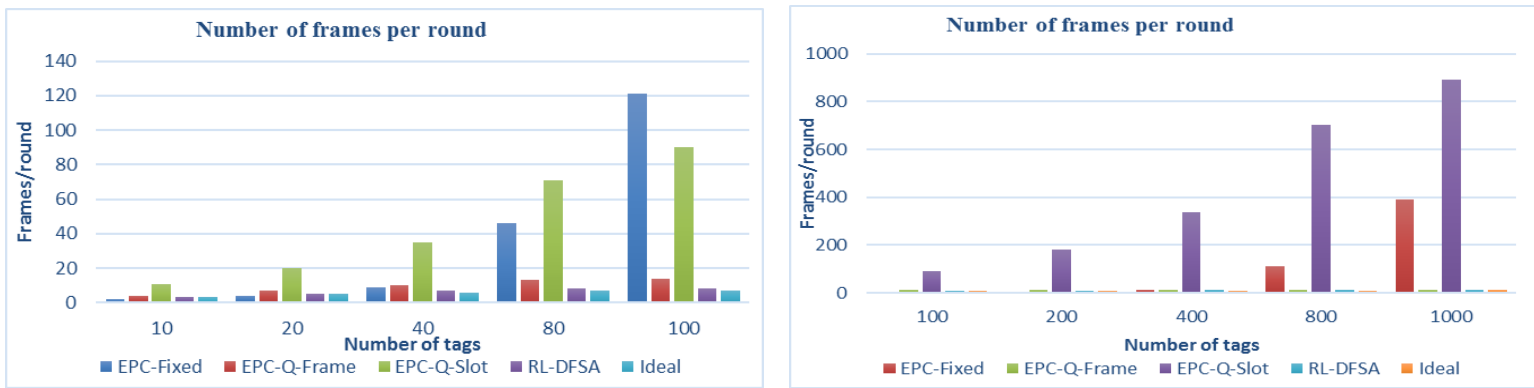

Figure 2. Average number of frames required per interrogation round for various algorithms

\section{CONCLUSION AND FUTURE WORKS}

Collision arbitration in RFID systems is crucial since collsion wastes precious energy of the battery-operated reader. This is not trivial since the tag population may vary from tens to thousands. Thus, in this work, we proposed RL-DFSA algorithm to address the collision and energy efficiency problems of RFID systems. RL-DFSA uses the Q-learning algorithm to select an optimal action which corresponds to a frame size among the available actions on each iteration based on the reward or punishment it got for selecting the previous action that leads it to be in its current state. The proposed algorithm was compared with EPC-Fixed, EPC-Q-Frame, EPC-Q-Slot, and Ideal algorithms in sparse and dense tag environments. The TSE metric of both the RL-DFSA and EPC-Q-Slot are almost identical while being very close to the performance of the Ideal algorithm. However, RL-DFSA achieved this near-optimal performance without the need for propagating excessive control messages as is the case with the EPC-Q-Slot algorithm Figure 2. Thus, RL-DFSA is energy efficient as compared to the EPC-Q-Slot since it is a known fact that the communication cost of a system is very high as compared to its computational cost.

\section{ACKNOWLEDGEMENTS}

The author would like to acknowledge the support from the Fundamental Research Grant Scheme (FRGS) under a grant number of FRGS/1/2017/ICT01/UNIMAP/02/1 from the Ministry of Higher Education Malaysia.

\section{REFERENCES}

[1] K. Finkenzeller, RFID Handbook: Fundamentals and Applications in Contactless Smart Cards, Radio Frequency Identification and near-Field Communication, Third Edition. Wiley, 2010.

[2] H. Cho, W. Lee, and Y. Baek, "LDFSA: A Learning-Based Dynamic Framed Slotted ALOHA for Collision Arbitration in Active RFID Systems," International Conference on Grid and Pervasive Computing. pp. 655-665, 2007.

[3] EPCglobal, "Specification for RFID Air Interface EPC TM Radio-Frequency Identity Protocols Class-1 Generation-2 UHF RFID," 2008.

[4] ISO/IEC, "Information technology -- Radio frequency identification for item management--Part 3: Parameters for air interface communications at 13,56 MHz," 2010.

[5] J. Wang, D. Wang, Y. Zhao, and T. Korhonen, "Fast anti-collision algorithms in RFID systems," Proc. - Int. Conf. Mob. Ubiquitous Comput. Syst. Serv. Technol. UBICOMM 2007, pp. 75-80, 2007.

[6] Y. Zhang and D. Zhao, "A new dynamic frame slotted ALOHA-algorithm for anti-collision in RFID systems," Proc. 2008 China-Japan Jt. Microw. Conf. CJMW 2008, pp. 502-504, 2008.

[7] F. C. Schoute, "Dvnamic Frame Length ALOHA," IEEE Trans. Commun., vol. 31, no. 4, pp. 565-568, 1983.

[8] S. Dhakal and S. Shin, "Precise-optimal frame length based collision reduction schemes for Frame Slotted Aloha RFID systems," KSII Trans. Internet Inf. Syst., vol. 8, no. 1, pp. 165-182, 2014.

[9] H. Vogt, "Efficient Object Identification with Passive RFID Tags," Pervasive Comput., pp. 98-113, 2002.

[10] L. Zhenzhen and E. Itamar, "RL-MAC: A QoS-Aware Reinforcement Learning based MAC Protocol for Wireless Sensor Networks," Int. J. Sens. Networks, vol. 1, no. 3, pp. 117-124, 2006. 
[11] GAORFID, “Android Based UHF Gen 2 RFID Handheld Data Terminal 246029.” pp. 1-3, 2018.

[12] Rapidradio, “UHF Handheld Reader RRUHFHH2.” pp. 1-2, 2018.

[13] M. Ghavamzadeh, H. J. Kappen, M. G. Azar, and R. Munos, "Speedy Q-Learning," Adv. Neural Inf. Process. Syst., pp. 2411-2419, 2011.

[14] P. Šolić, J. Radić, and N. Rožić, "Energy efficient tag estimation method for ALOHA-Based RFID Systems," IEEE Sens. J., vol. 14, no. 10, pp. 3637-3647, 2014.

[15] M. V. Bueno-Delgado and J. Vales-Alonso, "On the optimal frame-length configuration on real passive RFID systems," J. Netw. Comput. Appl., vol. 34, no. 3, pp. 864-876, 2011.

[16] A. Bachir, M. Dohler, T. Watteyne, I. Member, and I. S. Member, "MAC Essentials for Wireless Sensor Networks MAC Essentials for Wireless Sensor Networks,” Commun. Surv. \& Tutorials, vol. 12, no. 2, pp. 222-248, 2010.

\section{BIOGRAPHIES OF AUTHORS}

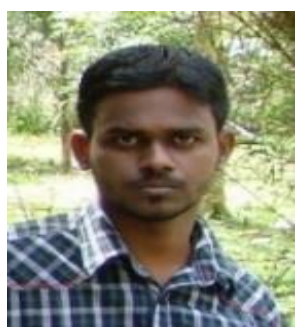

Murukesan Loganathan obtained his B. E (2012) and M. Sc (2015) in Mechatronic Engineering from the University Malaysia Perlis, Malaysia. Starting from October 2015, he is doing his Ph.D. in Computer Engineering under the supervision of Dr. Thennarasan Sabapathy at UniMAP. His research is mainly related on wireless sensor networks.

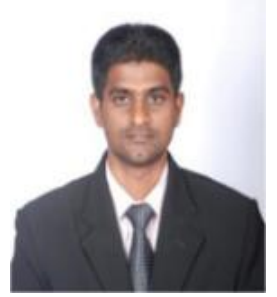

Thennarasan Sabapathy received the B.Eng. degree in electrical-telecommunication engineering from Universiti Teknologi Malaysia in 2007, the M.Eng. degree from Multimedia University, Malaysia, in 2011, and the Ph.D. degree in communication engineering from Universiti Malaysia Perlis in 2014. Currently he is a Senior Lecturer with the School of Computer and Communication Engineering. His current research interests include antenna, propagation and fuzzy logic for wireless communications.

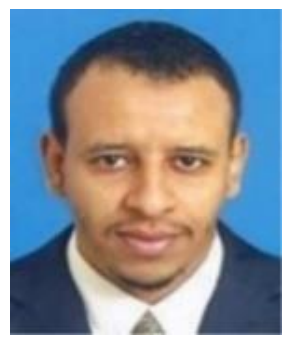

Mohamed Elobaid Elshaikh is a Senior Lecturer under School of Computer and Communication Engineering, University Malaysia Perlis, Malaysia. He received his Ph.D. in Computer Engineering from University Malaysia Perlis, M. Sc Electrical and Electronics Engineering from University Technology Petronas (UTP) and B. Sc Engineering Technology (Computer Engineering), University of Gezira, Sudan. His research mainly is on computer networking related.

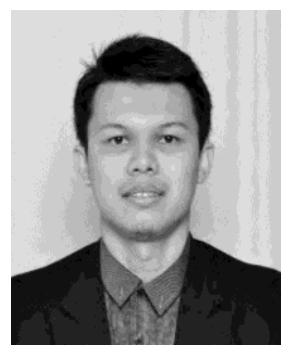

Mohamed Nasrun Osman was born Jitra, Malaysia, in 1987. He received the electrical engineering degree in telecommunication and the Ph.D. degree in electrical engineering from Universiti Teknologi Malaysia, in 2010 and 2016, respectively. He is currently a Senior Lecturer with Universiti Malaysia Perlis, Malaysia. His research interests include reconfigurable antenna design and wireless MIMO systems.

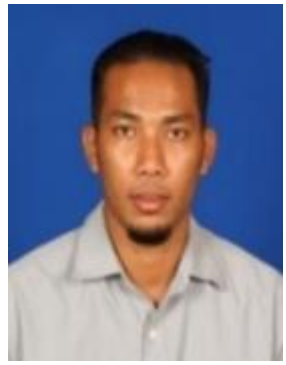

Rosemizi Abd Rahim received the B.Eng. degree in Electrical Engineering from the Universiti Teknologi Mara, Malaysia, in 2000 and the M.Sc. degree in Electronic System Design Engineering from the Universiti Sains Malaysia, in 2004. In 2013, he received the Ph.D. degree in Communication Engineering from the Universiti Malaysia Perlis. His research interest includes development of new sources of energy harvesting system and techniques, antenna design and microwave engineering. 